\title{
Bergamottin, a natural furanocoumarin abundantly present in grapefruit juice, suppresses the invasiveness of human glioma cells via inactivation of Rac1 signaling
}

\author{
WENZHENG LUO ${ }^{1}$, ZHENYU SONG $^{1}$, HONGWEI SUN ${ }^{1}$, JUNXIN LIANG ${ }^{1}$ and SHANSHAN ZHAO ${ }^{2}$ \\ Departments of ${ }^{1}$ Neurosurgery and ${ }^{2}$ Magnetic Resonance Imaging, \\ The First Affiliated Hospital of Zhengzhou University, Zhengzhou, Henan 450052, P.R. China
}

Received March 19, 2016; Accepted February 14, 2017

DOI: $10.3892 / \mathrm{ol} .2017 .7641$

\begin{abstract}
The aim of the present study was to explore the effect of bergamottin, a natural furanocoumarin obtained from grapefruit juice, on the invasiveness of human glioma cells. The results revealed that treatment with bergamottin for $48 \mathrm{~h}$ significantly inhibited wound-healing migration and Matrigel invasion of human glioma cells, compared with untreated cells $(\mathrm{P}<0.05)$. Bergamottin treatment caused a significant decrease in the expression and secretion of matrix metalloproteinase (MMP)-9 in glioma cells compared with untreated cells $(\mathrm{P}<0.05)$. A Rac1-GTP pull-down assay demonstrated that bergamottin-treated glioma cells had a significantly decreased level of active Rac1-GTP compared with untreated cells $(\mathrm{P}<0.05)$. However, bergamottin had no significant effect on cell division cycle 42 activity. Expression of constitutively activated Racl almost completely restored the migration and invasion of bergamottin-treated glioma cells. In addition, bergamottin-induced downregulation of MMP-9 was prevented by exogenous activated Rac1. The results of the present study demonstrated that bergamottin exhibits anti-invasive activity in human glioma cells through the inactivation of Rac1 and downregulation of MMP-9.
\end{abstract}

\section{Introduction}

Gliomas represent the most common type of primary tumors of the central nervous system (1). The highly invasive feature of malignant gliomas renders them refractory to surgery, radiation and chemotherapy (2). Despite improvement in treatment modalities, the median survival time for malignant gliomas is

Correspondence to: Professor Shanshan Zhao, Department of Magnetic Resonance Imaging, The First Affiliated Hospital of Zhengzhou University, 1 Jianshe East Road, Zhengzhou, Henan 450052, P.R. China

E-mail: drshanshanzhao@qq.com

Key words: glioma, invasiveness, phytochemical, Rac1 signaling only between 1 and 2 years $(3,4)$. Therefore, it is of importance to develop novel effective therapies against invasive gliomas.

Rho GTPases are a family of small signaling G-proteins that perform critical roles in cancer cell migration, invasion and metastasis (5). They act as molecular switches through cycling between an inactive GDP-bound and active GTP-bound form. Racl is one of the most studied Rho GTPase family members (5). Several lines of evidence indicate that Rac1 activation contributes to glioma cell invasion (6-8). Nakada et al (6) reported that the ephrin-B3 ligand facilitates the invasion of glioma cells through activation of Rac1. Tumor necrosis factor receptor superfamily member 19 (TNFRSF19) overexpression has been found to activate Racl signaling to drive glioma cell invasion and migration, and inactivation of Rac1 may abrogate TNFRSF19-induced glioma cell invasion (8). ZINC69391, a specific Rac1 inhibitor, has been reported to exhibit anti-invasive activity on glioma cells (9). These studies indicate a pivotal role for Rac1 signaling in glioma invasion. Matrix metalloproteinases (MMPs), which are important regulators of extracellular matrix degradation, have been documented to be implicated in Rac1-induced cell invasion (10).

Bergamottin, a natural furanocoumarin abundantly present in grapefruit juice, has been demonstrated to exhibit anticancer effects in a variety of human cancers, including chronic myelogenous leukemia (11), multiple myeloma (12), skin cancer (13) and breast cancer (14). It has been reported that bergamottin enhances tumor necrosis factor-induced apoptosis in human chronic myelogenous leukemia via inactivation of nuclear factor- $\kappa \mathrm{B}$ signaling (11). Kim et al (12) reported that bergamottin exhibits chemosensitizing activity in multiple myeloma cells via inhibition of the signal transducer and activator of transcription 3 signaling pathway. Hwang et al (15) demonstrated that bergamottin may prevent phorbol 12-myristate 13-acetate-induced tumor cell invasion via downregulation of MMP-9 expression. Despite these studies, relatively little is known about the effect of bergamottin in human glioma.

Therefore, in the present study, the potential anticancer effect of bergamottin on human glioma cells was explored. Considering the importance of Racl in glioma invasion, the involvement of Racl in the action of bergamottin was also investigated. 
A<smiles>CC(C)=CCC/C(C)=C/COc1c2ccoc2cc2oc(=O)ccc12</smiles>

C

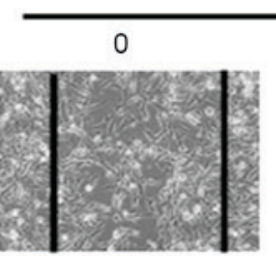

U251

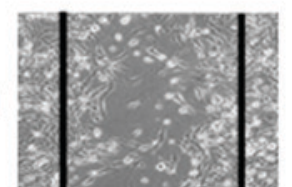

Bergamottin ( $\mu \mathrm{M})$
2
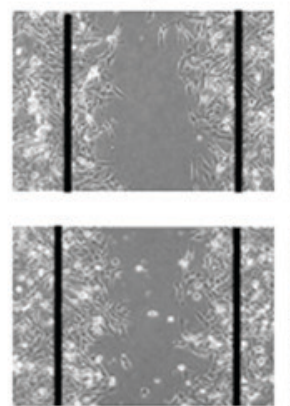

B

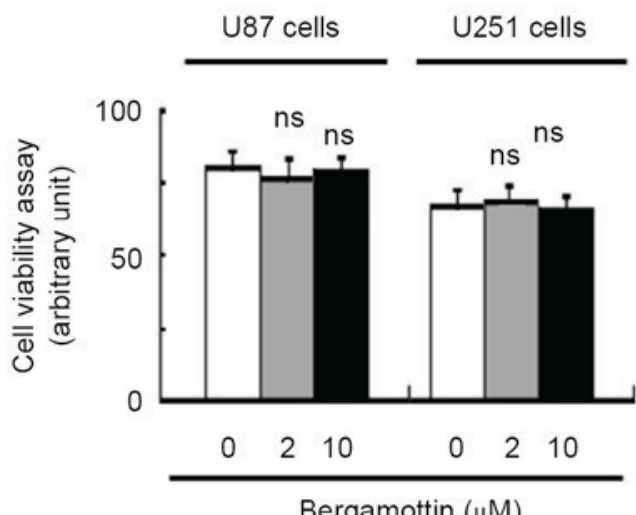

Bergamottin $(\mu \mathrm{M})$

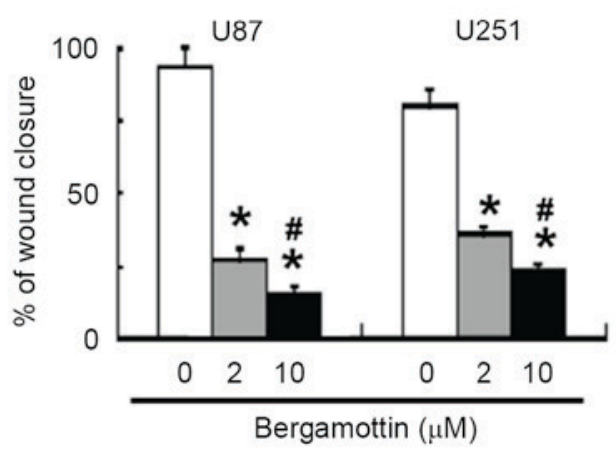

Figure 1. Bergamottin inhibits the migration of glioma cells. (A) Chemical structure of bergamottin. (B) Glioma cells in serum-free medium were treated with the indicated concentrations of bergamottin for $48 \mathrm{~h}$ and tested for cell viability. Quantitative data from three independent experiments are presented. (C) Cells were treated with different concentrations of bergamottin for $48 \mathrm{~h}$ and subjected to wound-healing assay. Images are representative from one experiment and were captured $48 \mathrm{~h}$ after scratching (magnification, $\mathrm{x} 40$ ). The mean percentages ( \pm standard error) of three independent experiments performed with two replicates are presented. " $\mathrm{P}<0.05$ vs. untreated cells; ${ }^{\#} \mathrm{P}<0.05$ vs. $2 \mu \mathrm{M}$ bergamottin. NS, no significance when compared with control cells.

\section{Materials and methods}

Cell culture and treatment. Human U87 and U251 glioma cells were purchased from the American Type Culture Collection (Manassas, VA, USA). Cells were maintained in Dulbecco's modified Eagle's medium (DMEM; Invitrogen; Thermo Fisher Scientific, Inc., Waltham, MA, USA) supplemented with $10 \%$ heat-inactivated fetal bovine serum (FBS) and $1 \%$ penicillin $(100 \mathrm{IU} / \mathrm{ml}) /$ streptomycin $(100 \mu \mathrm{g} / \mathrm{ml}$; all from Invitrogen; Thermo Fisher Scientific, Inc.), at $37^{\circ} \mathrm{C}$ in a humidified atmosphere containing $5 \% \mathrm{CO}_{2}$.

Bergamottin ( $\geq 96.9 \%$ in purity; Fig. $1 \mathrm{~A})$ was purchased from ChromaDex (Irvine, CA, USA). The cells were incubated with 2 and $10 \mu \mathrm{M}$ bergamottin for $48 \mathrm{~h}$ at $37^{\circ} \mathrm{C}$ and collected for subsequent analyses.

Plasmid transfection. A constitutively active Rac1 construct (pcDNA3-EGFP-Rac1-Q61L) was purchased from Addgene, Inc. (Cambridge, MA, USA). Transient transfection of pcDNA3-EGFP-Rac1-Q61L or empty vector was performed using Fugene reagent (Roche Diagnostics, Basel, Switzerland), according to the manufacturer's protocol. At $24 \mathrm{~h}$ post-transfection, cells were exposed to bergamottin for an additional $48 \mathrm{~h}$ and examined for cell migration, invasion and MMP-9 expression. To estimate transfection efficiency, parallel cell cultures were transfected with a green fluorescent protein (GFP)-encoding plasmid (pEGFP-C1; Clontech Laboratories, Inc., Mountain View, CA, USA). In the present study, transfection efficiency was $>85 \%$.

Cell viability assay. The cells in serum-free medium were seeded at $5 \times 10^{3}$ cells/well in 96 -well plates and treated with bergamottin for $48 \mathrm{~h}$. Cell proliferation was assessed using the Cell Titer 96 aqueous non-radioactive cell proliferation assay kit (Promega Corporation, WI, USA), according to the manufacturer's protocol. Absorbance was read at $490 \mathrm{~nm}$ using a microplate reader.

Wound-healing assay. Cells were seeded in 6-well plates and grown to $100 \%$ confluence. A scratch wound was made in the cell monolayer using a $10 \mu 1$ pipette tip. Detached and damaged cells were carefully washed with PBS. The adherent cells were then cultured in DMEM containing $1 \%$ FBS with or without bergamottin. Following incubation for $48 \mathrm{~h}$ at $37^{\circ} \mathrm{C}$, images of cells were captured using a light microscope (DM6000 B; Leica Microsystems GmbH, Wetzlar, Germany) in 5 random fields (magnification, $x 40$ ). The percentage of wound closure was calculated as follows: [(Initial wound area-post-migration area)/initial wound area] $\times 100$. 
A

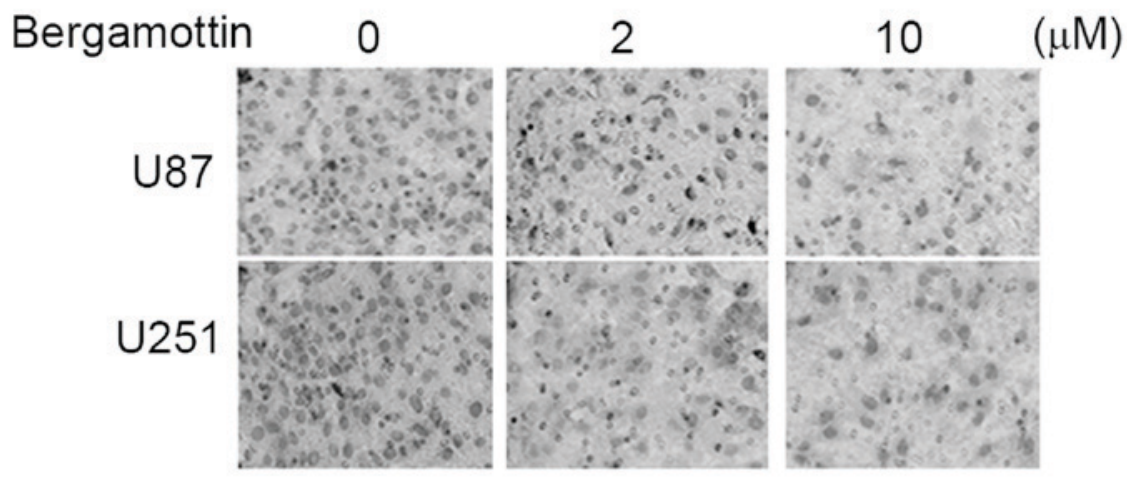

B

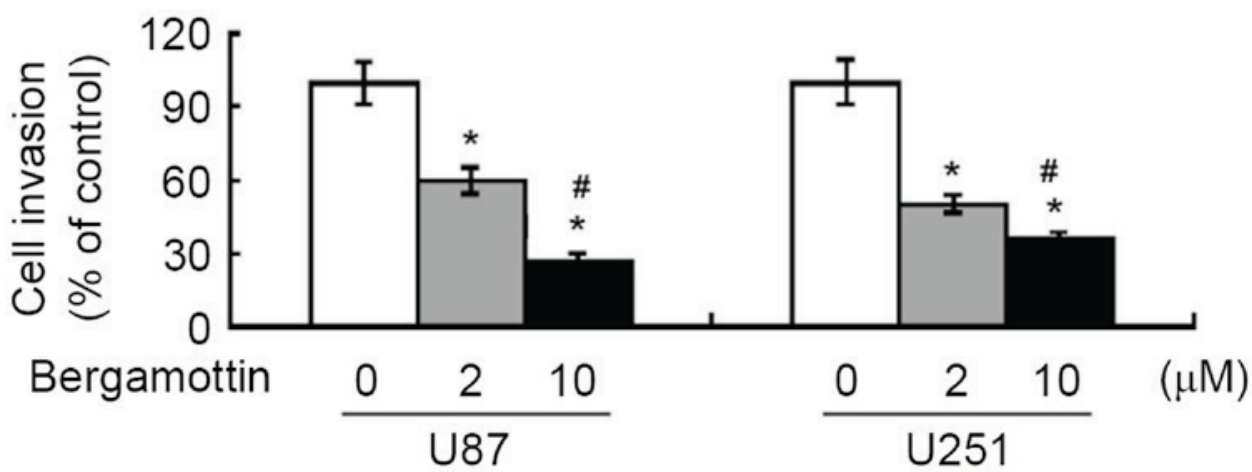

Figure 2. Bergamottin suppresses the invasion of glioma cells. Cells were treated with the indicated concentrations of bergamottin for $48 \mathrm{~h}$ and the invasiveness was determined using a Matrigel Transwell assay. (A) Representative images of invasion assay at x200 magnification. (B) Quantitative data from three independent experiments performed with two replicates are presented. ${ }^{*} \mathrm{P}<0.05$ vs. untreated cells; ${ }^{\#} \mathrm{P}<0.05$ vs. $2 \mu \mathrm{M}$ bergamottin.

Transwell invasion assay. Transwell chambers in 24-well plates were coated with Matrigel (BD Biosciences, Franklin Lakes, NJ, USA) in DMEM and incubated at $37^{\circ} \mathrm{C}$ for $45 \mathrm{~min}$ to allow the gel to solidify. The lower chambers were filled with DMEM with $10 \%$ FBS as a chemoattractant. The upper chambers were seeded with $2 \times 10^{4}$ cells/well in serum-free DMEM containing 2 or $10 \mu \mathrm{M}$ bergamottin. Following incubation for $48 \mathrm{~h}$ at $37^{\circ} \mathrm{C}$, non-invading cells (upper chamber) were gently removed with a cotton swab. Invading cells (lower chamber) were fixed with $4 \%$ paraformaldehyde (Sigma-Aldrich; Merck KGaA, Darmstadt, Germany), stained with $0.2 \%$ crystal violet (Sigma-Aldrich; Merck KGaA) and counted under a light microscope (DM6000 B; Leica Microsystems $\mathrm{GmbH}$ ). A total of 10 random microscopic fields (magnification, x200) were examined for each well.

Western blot analysis of MMP-9 protein. Following treatment, cells were collected and lysed in ice-cold radioimmunoprecipitation assay buffer (Cell Signaling Technology, Inc., Danvers, MA, USA) containing $50 \mathrm{mM}$ sodium fluoride, $1 \mathrm{mM}$ phenylmethylsulfonyl fluoride, and $10 \mu \mathrm{g} / \mathrm{ml}$ aprotinin and leupeptin (Sigma-Aldrich; Merck KGaA). The lysates were clarified and quantified using the Bradford Protein assay (Bio-Rad Laboratories, Inc., Hercules, CA, USA), according to the manufacturer's protocol.Equal amounts of total protein ( $40 \mu \mathrm{g}$ per lane) were resolved by $10 \%$ SDS-PAGE and transferred onto nitrocellulose membranes. Following blocking with $10 \%$ fat-free milk, the membrane was incubated with rabbit anti-MMP-9 antibody (1:500; cat. no. sc-393859) or anti- $\beta$-actin antibody $(1: 1,000$; cat. no. sc-130301; Santa Cruz Biotechnology, Inc., Dallas, TX,
USA) at $4^{\circ} \mathrm{C}$ overnight, followed by incubation at room temperature for $1 \mathrm{~h}$ with horseradish peroxidase-conjugated secondary antibody (1:3,000; cat. no. sc-2005; Santa Cruz Biotechnology, Inc.). Protein bands were visualized using enhanced chemiluminescence (GE Healthcare Life Sciences, Chalfont, UK) and quantified using Quantity One software (version 4.6.2; Bio-Rad Laboratories Inc.).

Rho GTPase activity assays. U87 and U251 cells were treated for $48 \mathrm{~h}$ with or without bergamottin, and the Rac1 Activation assay kit was performed according to the manufacturer's protocol (Cell Biolabs, Inc., San Diego, CA, USA). Following treatment, cells were lysed in tissue culture plates and cell lysates were clarified by centrifugation at $12,000 \mathrm{x} \mathrm{g}$ for $10 \mathrm{~min}$ at $4^{\circ} \mathrm{C}$. The protein concentration was quantified using the Bradford protein assay. Lysates (50 $\mu \mathrm{g} /$ lane) were diluted and incubated for $1 \mathrm{~h}$ with glutathione transferase-fusion proteins containing the p21-binding domain of p21-activated protein kinase 1 . Bound complexes were washed and subjected to western blot analysis using mouse monoclonal antibody anti-Rac1 (1:300; cat. no., 610650; BD Biosciences) or anti-cell division cycle (Cdc) 42 (1:300; cat. no., 610929; BD Biosciences). The intensity of immunoreactive signals was quantified using Quantity One software (version 4.6.2; Bio-Rad Laboratories, Inc.).

ELISA. Cells were treated with bergamottin for $48 \mathrm{~h}$ and the supernatants were collected and centrifuged at 12,000 x g for $10 \mathrm{~min}$ at $4^{\circ} \mathrm{C}$. The protein concentrations of MMP-9 in 


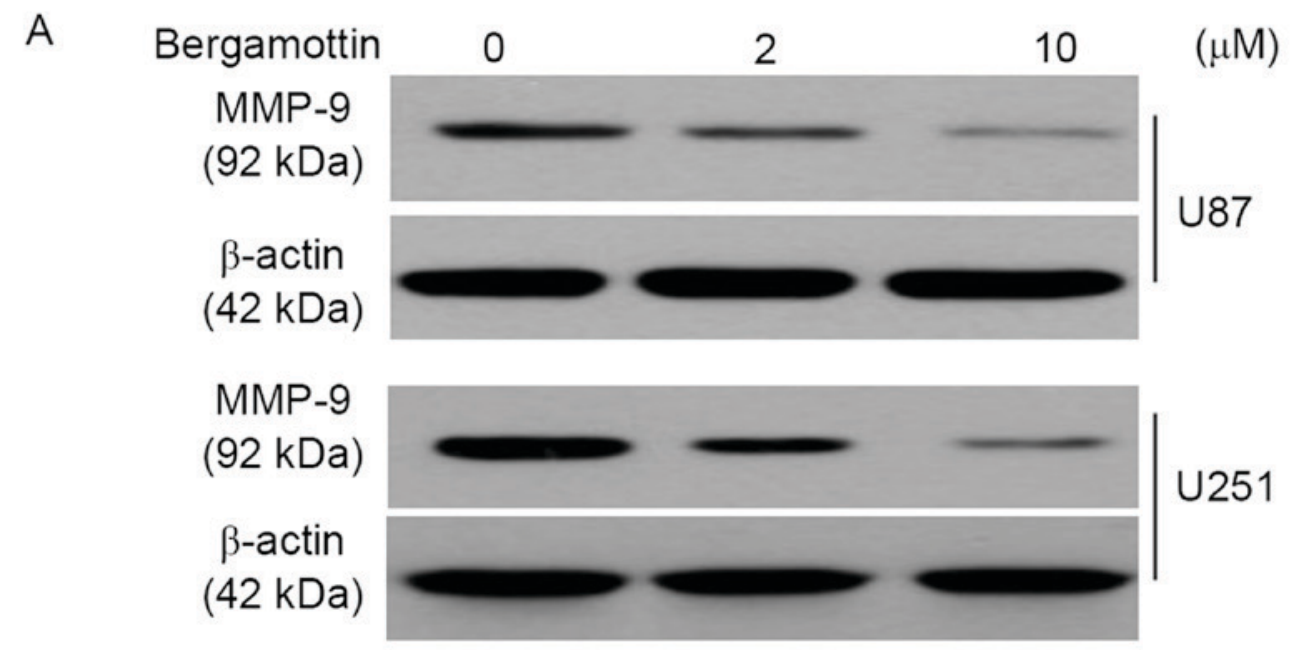

B
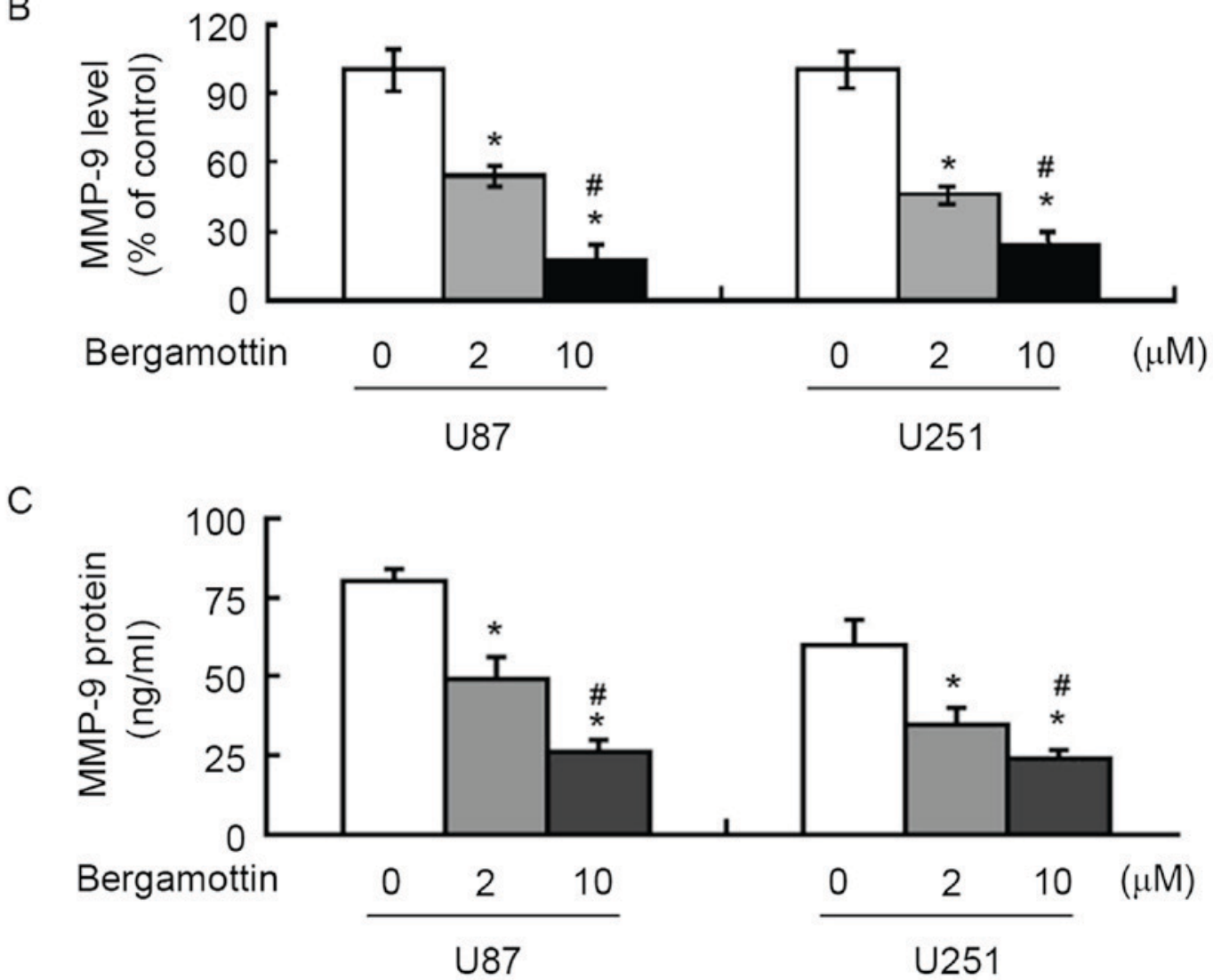

Figure 3. Bergamottin treatment impairs the expression and secretion of MMP-9. Cells were treated with the indicated concentrations of bergamottin for $48 \mathrm{~h}$ and examined for MMP-9 expression. (A) Western blot analysis of MMP-9 protein. Blots are representative of three independent experiments. (B) Quantitative data from three independent experiments performed with three replicates are presented. (C) ELISA analysis of MMP-9 levels in the supernatants from treated cells. Quantitative data from three independent experiments performed with three replicates are presented. ${ }^{*} \mathrm{P}<0.05$ vs. untreated cells; ${ }^{*} \mathrm{P}<0.05$ vs. $2 \mu \mathrm{M}$ bergamottin. MMP-9, matrix metalloproteinase-9.

the supernatants were determined using the human MMP-9 Quantikine ELISA kit according to the manufacturer's protocol (R\&D Systems, Inc., Minneapolis, MN, USA).

Statistical analysis. All data are presented as the mean \pm standard error. The significance of differences among groups was determined using one-way analysis of variance followed by Tukey's test. $\mathrm{P}<0.05$ was considered to indicate a statistically significant difference.

\section{Results}

Bergamottin inhibits migration and invasion of human glioma cells. Exposure to bergamottin $<10 \mu \mathrm{M}$ in serum-free medium had no significant effect on the viability of U87 and U251 cells, compared with control cells (Fig. 1B). Wound-healing assays demonstrated that bergamottin suppressed the migration of U87 and U251 cells into the wound in cell monolayers (Fig. 1C). Quantification analysis indicated that the percentage of wound 
A

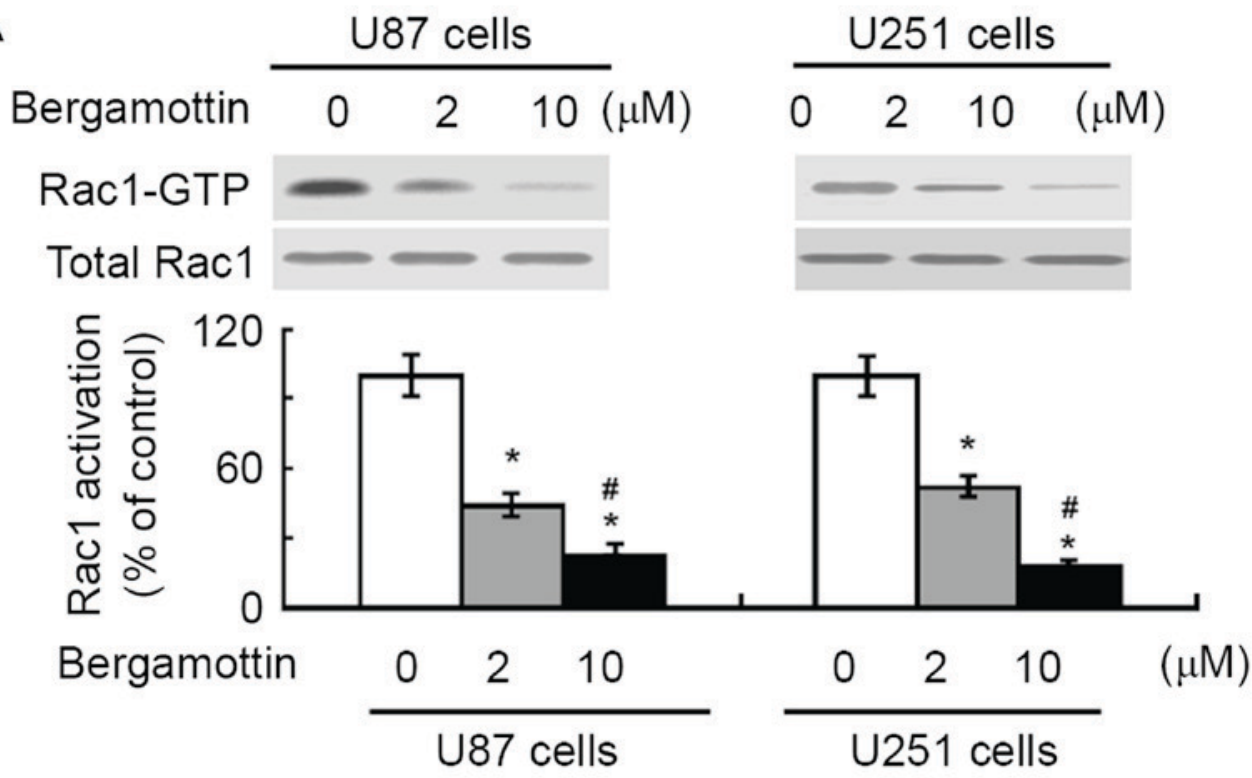

B

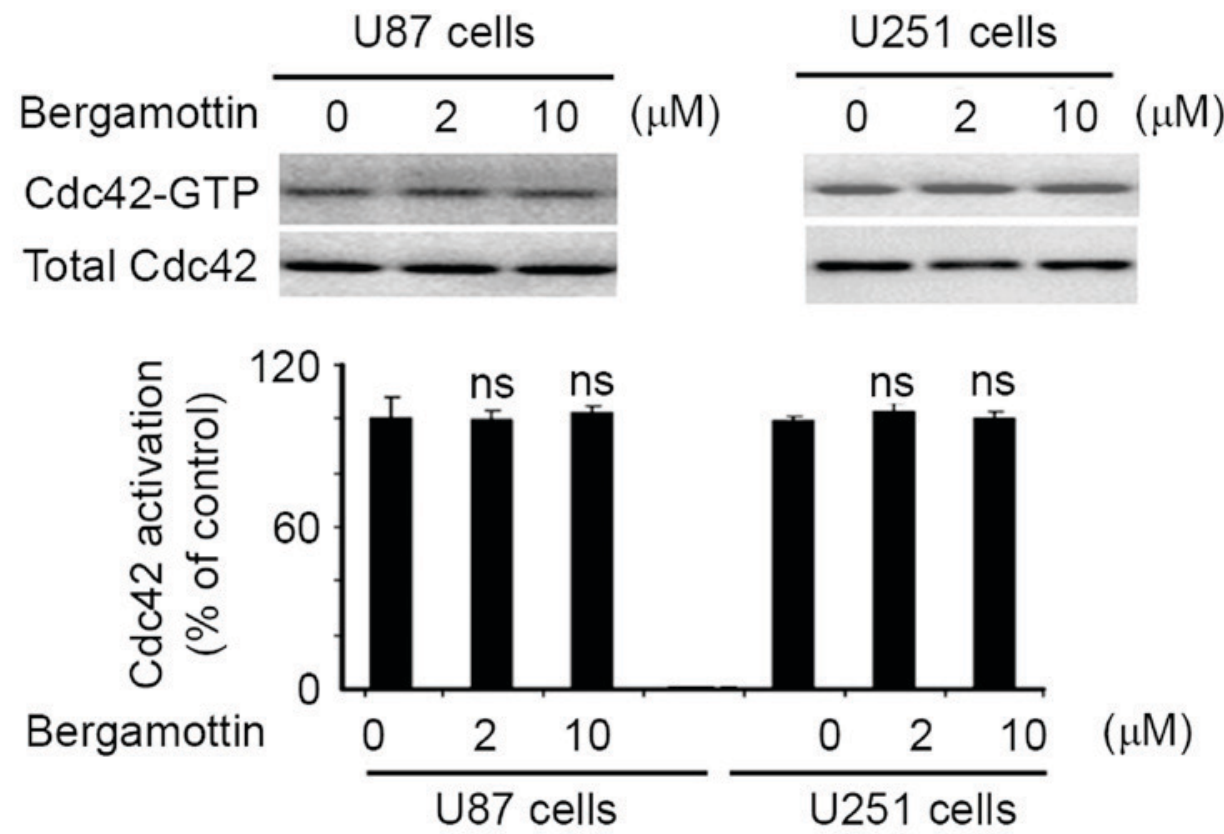

Figure 4. Bergamottin regulates Racl activation. (A) Measurement of the levels of Rac1-GTP in glioma cells with the indicated treatments using GST-PBD pull-down assay and western blot analysis using anti-Rac1 antibody. Band intensity of Rac1-GTP was normalized against total Rac1. (B) Detection of Cdc42 activity using the GST-PBD pull-down assay and western blot analysis using anti-Cdc42 antibody. Quantitative data from three independent experiments performed with three replicates are presented. ${ }^{*} \mathrm{P}<0.05$ vs. untreated cells; ${ }^{*} \mathrm{P}<0.05 \mathrm{vs} .2 \mu \mathrm{M}$ bergamottin. ns, not significant compared with control cells. Cdc42, cell division cycle 42; GST, glutathione transferase; PBD, p21-binding domain.

closure was significantly decreased in bergamottin-treated cells compared with untreated control cells $(\mathrm{P}<0.05)$. The invasive ability of glioma cells was assessed using Matrigel-coated Transwell assays. Following bergamottin treatment for $48 \mathrm{~h}$, the number of invading cells was decreased between 40 and $70 \%$ in comparison with control cells $(\mathrm{P}<0.05$; Fig. 2).

Bergamottin suppresses the expression and secretion of MMP-9. The effects of bergamottin on the expression and secretion of MMP-9 were then assessed. Bergamottin at 2 and $10 \mu \mathrm{M}$ decreased MMP-9 protein expression in U87 and U251 cells (Fig. 3A), which was determined to be significant ( $\mathrm{P}<0.05$; Fig. 3B). ELISA confirmed that the supernatants from bergamottin-treated cells expressed significantly decreased levels of MMP-9 compared with those from control cells $(\mathrm{P}<0.05$; Fig. 3C).

Bergamottin interferes with the activation of Racl. To examine the effect of bergamottin on Rac1 activation, Rac1-GTP pull-down assays were performed in U87 and U251 cells treated with or without bergamottin. Bergamottin treatment caused a significant decrease decline in the level of active Rac1-GTP, compared with untreated cells $(\mathrm{P}<0.05$; Fig. 4A). However, no 
A

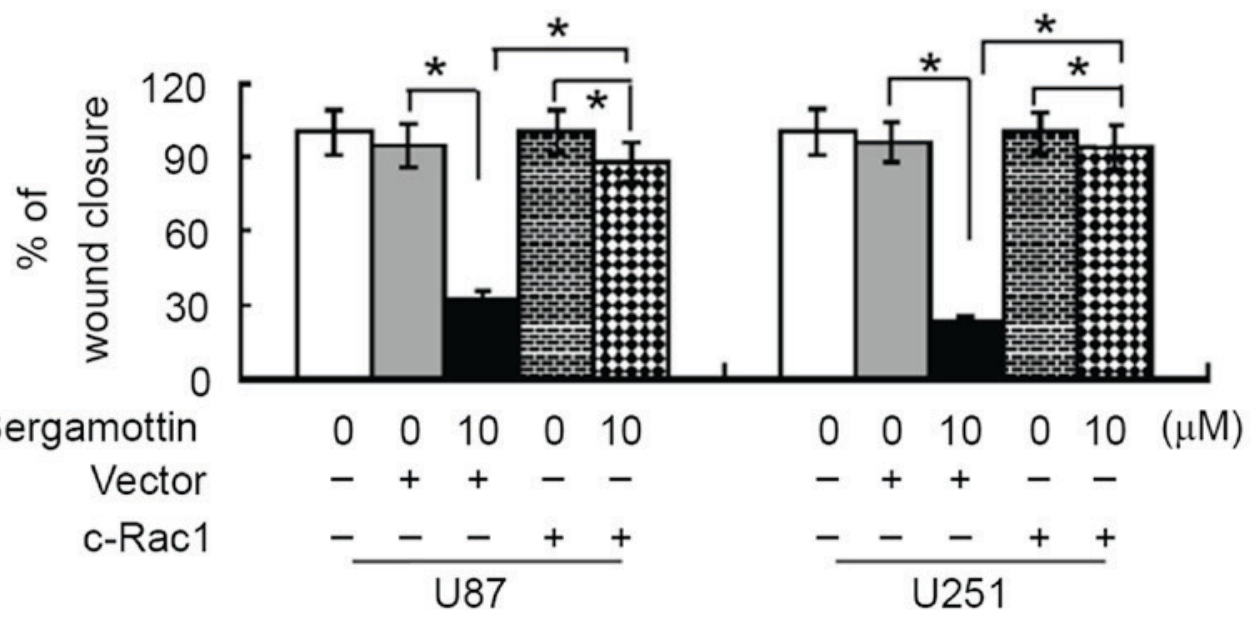

B
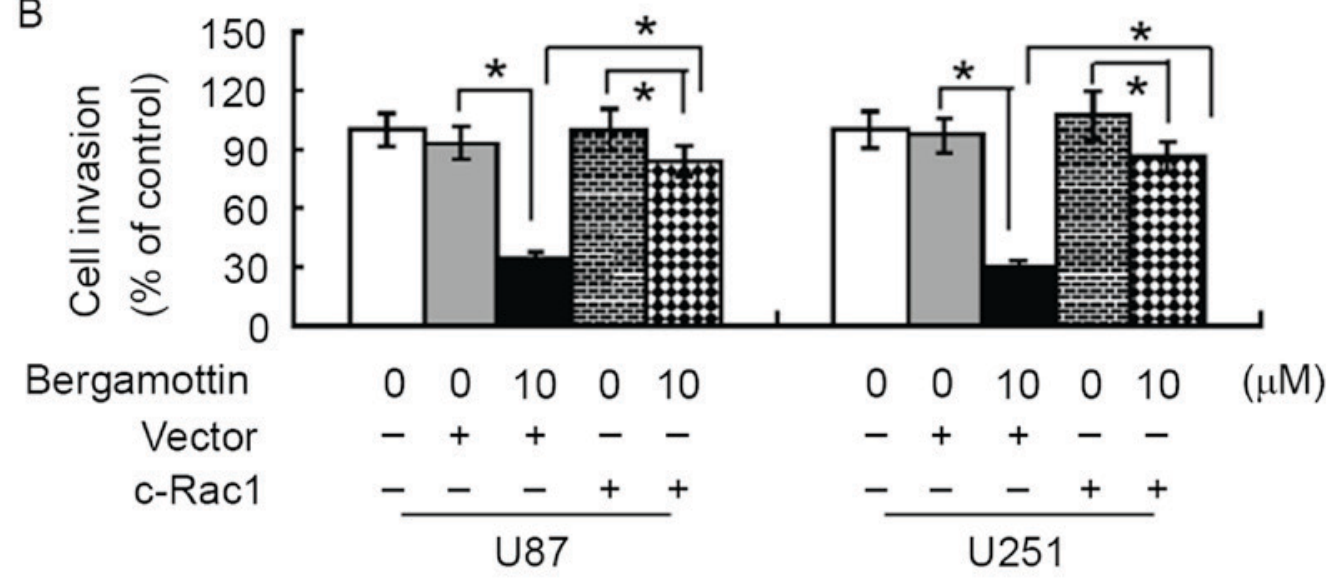

C

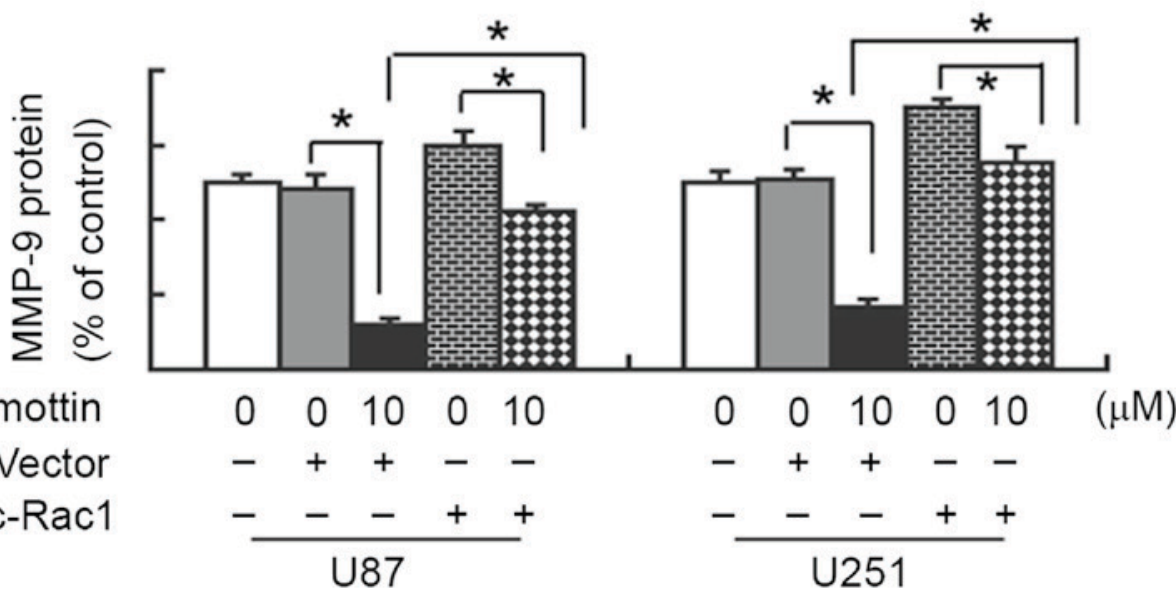

Figure 5. Rac1 inactivation is involved in the anti-invasive activity of bergamottin. Cells were pre-transfected with constitutively activated Rac1 or empty vector prior to treatment with $10 \mu \mathrm{M}$ bergamottin. (A) Cell migration and (B) cell invasion were assessed using a wound-healing assay and a Matrigel Transwell invasion assay, respectively. (C) Western blot analysis of MMP-9 protein levels. Quantitative data from three independent experiments performed with three replicates are presented. ${ }^{*} \mathrm{P}<0.05$. MMP-9, matrix metalloproteinase-9.

change in Cdc42 activity was detected (Fig. 4B). These results indicated that bergamottin is specific for inhibition of Rac1.

Racl inactivation is involved in the anti-invasive activity of bergamottin. To validate whether the anti-invasive effect of bergamottin is mediated through Rac1 inactivation, glioma cells were pre-transfected with constitutively activated Racl or empty vector prior to exposure to $10 \mu \mathrm{M}$ bergamottin, and cell migration and invasion were assessed. Notably, the presence of constitutively activated Rac1 almost completely restored the migration and invasion of bergamottin-treated U87 and U251 cells (Fig. 5A and B). In addition, bergamottin-induced suppression of MMP-9 protein expression was significantly prevented by exogenous activated Rac1 ( $\mathrm{P}<0.05$; Fig. 5C). 


\section{Discussion}

There is growing interest in the application of plant phytochemicals as anticancer agents (16-18). Racoma et al (17) reported that thymoquinone, a bioactive compound of the Nigella sativa seed oil, exerts inhibitory effects on the clonogenicity of glioblastoma cells, but not of normal human astrocytes. Quercetin (a dietary flavonoid) has been demonstrated to promote apoptotic death in human glioma cells (18). Bergamottin has been reported to induce an apoptotic response in tumor cells $(11,12)$. To the best of our knowledge, the present study provides the first evidence for the anticancer potential of bergamottin in human glioma. The results of the present study demonstrated that bergamottin treatment causes a significant suppression of the migration and invasion of human glioma cells. The anti-invasive activity of bergamottin has also been described previously (15). These results indicated that bergamottin affects multiple aspects of cancer development and progression.

Mechanistically, it was revealed that bergamottin treatment significantly impaired the activation of Rac1 and downregulated the expression of MMP-9. Rac1 has been identified as a key regulator of cancer cell invasion (6-8). C6 glioma cells with high Rac1 activity possess increased invasiveness compared with those with low Racl activity (19), indicating a favorable role for Rac1 in glioma invasion. Targeting Rac1 abrogates cancer progression and metastasis $(20,21)$. Rac1 small-molecule inhibitors (ZINC69391 and 1A-116) have been documented to decrease cell proliferation, trigger apoptotic death and inhibit cell migration and invasion in malignant glioma cells (9). These studies indicated that Rac1 is a potential target for controlling cancer invasion and metastasis. To confirm the role of Rac1 in bergamottin-mediated anti-invasive effects, active Rac1 was constitutively expressed in glioma cells and changes in the invasiveness of glioma cells were evaluated. The results of the present study demonstrated that U87 and U251 cells with constitutively activated Rac1 maintained their invasiveness in the presence of bergamottin, suggesting that the anti-invasive activity of bergamottin in glioma cells is largely mediated through inactivation of Rac1.

The pro-invasive activity of Racl is associated with enhanced expression and secretion of MMPs (22-24). Zhang et al (23) reported that extracellular ATP facilitates the invasion of prostate cancer cells through the activation of Racl and Cdc42 and the upregulation of MMP-3 and MMP-13. The results of the present study demonstrated that enforced expression of activated Rac1 prevented the suppression of MMP-9 expression in glioma cells by bergamottin. MMP-9 performs a critical role in glioma cell invasion. Downregulation of MMP-3 and MMP-9 has been demonstrated to contribute to inhibition of glioma cell invasion by glycitein, a bacterial metabolite of the isoflavone glycitin (25). Targeting MMP-9 via small interfering RNA leads to decreased invasiveness of glioma cells (26). Taken together, bergamottin-mediated suppression of glioma cell invasion is associated with inactivation of Rac1 and a subsequent decrease in MMP-9 expression. However, the signaling pathways involved in bergamottin-induced inactivation of Racl remain unclear.

To the best of the knowledge of the authors, the present study provided the first evidence that bergamottin has anti-invasive activity in human glioma cells, which is primarily associated with inhibition of Racl activation and downregulation of
MMP-9. These results indicated that bergamottin has therapeutic potential for the treatment of metastatic glioma.

\section{References}

1. Altieri R, Agnoletti A, Quattrucci F, Garbossa D, Calamo Specchia FM, Bozzaro M, Fornaro R, Mencarani C, Lanotte M, Spaziante R and Ducati A: Molecular biology of gliomas: Present and future challenges. Transl Med UniSa 10: 29-37, 2014.

2. Siebzehnrubl FA, Silver DJ, Tugertimur B, Deleyrolle LP, Siebzehnrubl D, Sarkisian MR, Devers KG, Yachnis AT, Kupper MD, Neal D, et al: The ZEB1 pathway links glioblastoma initiation, invasion and chemoresistance. EMBO Mol Med 5: 1196-1212, 2013.

3. Wen PY and Kesari S: Malignant gliomas in adults. N Engl J Med 359: 492-507, 2008.

4. Tanase CP, Enciu AM, Mihai S, Neagu AI, Calenic B and Cruceru ML: Anti-cancer therapies in high grade gliomas. Curr Proteomics 10: 246-260, 2013.

5. Fortin Ensign SP, Mathews IT, Symons MH, Berens ME and Tran NL: Implications of Rho GTPase signaling in glioma cell invasion and tumor progression. Front Oncol 3: 241, 2013.

6. Nakada M, Drake KL, Nakada S, Niska JA and Berens ME: Ephrin-B3 ligand promotes glioma invasion through activation of Rac1. Cancer Res 66: 8492-8500, 2006.

7. Hu B, Shi B, Jarzynka MJ, Yiin JJ, D'Souza-Schorey C and Cheng SY: ADP-ribosylation factor 6 regulates glioma cell invasion through the IQ-domain GTPase-activating protein 1-Rac1-mediated pathway. Cancer Res 69: 794-801, 2009.

8. Paulino VM, Yang Z, Kloss J, Ennis MJ, Armstrong BA, Loftus JC and Tran NL: TROY (TNFRSF19) is overexpressed in advanced glial tumors and promotes glioblastoma cell invasion via Pyk2-Rac1 signaling. Mol Cancer Res 8: 1558-1567, 2010.

9. Cardama GA, Gonzalez N, Ciarlantini M, Gandolfi Donadío L, Comin MJ, Alonso DF, Menna PL and Gomez DE: Proapoptotic and antiinvasive activity of Racl small molecule inhibitors on malignant glioma cells. Onco Targets Ther 7: 2021-2033, 2014.

10. Shin I, Kim S, Song H, Kim HR and Moon A: H-Ras-specific activation of Rac-MKK3/6-p38 pathway: Its critical role in invasion and migration of breast epithelial cells. J Biol Chem 80: 14675-14683, 2005.

11. Kim SM, Lee EJ, Lee JH, Yang WM, Nam D, Lee JH, Lee SG, Um JY, Shim BS and Ahn KS: Simvastatin in combination with bergamottin potentiates TNF-induced apoptosis through modulation of NF- $\kappa \mathrm{B}$ signalling pathway in human chronic myelogenous leukaemia. Pharm Biol 54: 2050-2060, 2016.

12. Kim SM, Lee JH, Sethi G, Kim C, Baek SH, Nam D, Chung WS Kim SH, Shim BS and Ahn KS: Bergamottin, a natural furanocoumarin obtained from grapefruit juice induces chemosensitization and apoptosis through the inhibition of STAT3 signaling pathway in tumor cells. Cancer Lett 354: 153-163, 2014.

13. Kleiner HE, Vulimiri SV, Reed MJ, Uberecken A and DiGiovanni J: Role of cytochrome P450 1a1 and 1b1 in the metabolic activation of 7,12-dimethylbenz[a]anthracene and the effects of naturally occurring furanocoumarins on skin tumor initiation. Chem Res Toxicol 15: 226-235, 2002.

14. Kleiner HE, Reed MJ and DiGiovanni J: Naturally occurring coumarins inhibit human cytochromes P450 and block benzo[a] pyrene and 7,12-dimethylbenz[a]anthracene DNA adduct formation in MCF-7 cells. Chem Res Toxicol 16: 415-422, 2003.

15. Hwang YP, Yun HJ, Choi JH, Kang KW and Jeong HG: Suppression of phorbol-12-myristate-13-acetate-induced tumor cell invasion by bergamottin via the inhibition of protein kinase Cdelta/p38 mitogen-activated protein kinase and JNK/nuclear factor-kappaB-dependent matrix metalloproteinase- 9 expression. Mol Nutr Food Res 54: 977-990, 2010.

16. Øverby A, Zhao CM and Chen D: Plant phytochemicals: Potential anticancer agents against gastric cancer. Curr Opin Pharmacol 19: 6-10, 2014.

17. Racoma IO, Meisen WH, Wang QE, Kaur B and Wani AA: Thymoquinone inhibits autophagy and induces cathepsin-mediated, caspase-independent cell death in glioblastoma cells. PLoS One 8: e72882, 2013

18. Pan HC, Jiang Q, Yu Y, Mei JP, Cui YK and Zhao WJ: Quercetin promotes cell apoptosis and inhibits the expression of MMP-9 and fibronectin via the AKT and ERK signalling pathways in human glioma cells. Neurochem Int 80: 60-71, 2015. 
19. Yukinaga H, Shionyu C, Hirata E, Ui-Tei K, Nagashima T, Kondo S, Okada-Hatakeyama M, Naoki $\mathrm{H}$ and Matsuda M: Fluctuation of Racl activity is associated with the phenotypic and transcriptional heterogeneity of glioma cells. J Cell Sci 127: $1805-1815,2014$

20. Cao X, Zhang L, Shi Y, Sun Y, Dai S, Guo C,Zhu F, Wang Q, Wang J, Wang X, et al: Human tumor necrosis factor (TNF)-alpha-induced protein 8-like 2 suppresses hepatocellular carcinoma metastasis through inhibiting Rac1. Mol Cancer 12: 149, 2013.

21. Zhao H, Dong T, Zhou H, Wang L, Huang A, Feng B, Quan Y, Jin R, Zhang W, Sun J, et al: miR-320a suppresses colorectal cancer progression by targeting Rac1. Carcinogenesis 35: 886-895, 2014

22. Zhuge $\mathrm{Y}$ and $\mathrm{Xu}$ J: Rac1 mediates type I collagen-dependent MMP-2 activation. Role in cell invasion across collagen barrier. J Biol Chem 276: 16248-16256, 2001.

23. Zhang Y, Gong LH, Zhang HQ, Du Q, You JF, Tian XX and Fang WG: Extracellular ATP enhances in vitro invasion of prostate cancer cells by activating Rho GTPase and upregulating MMPs expression. Cancer Lett 293: 189-197, 2010.
24. Chen QY, Zheng Y, Jiao DM, Chen FY, Hu HZ, Wu YQ, Song J, Yan J, Wu LJ and Lv GY: Curcumin inhibits lung cancer cell migration and invasion through Racl-dependent signaling pathway. J Nutr Biochem 25: 177-185, 2014

25. Lee EJ, Kim SY, Hyun JW, Min SW, Kim DH and Kim HS: Glycitein inhibits glioma cell invasion through down-regulation of MMP-3 and MMP-9 gene expression. Chem Biol Interact 185: $18-24,2010$

26. Gondi CS, Lakka SS, Dinh DH, Olivero WC, Gujrati M and Rao JS: Downregulation of uPA, uPAR and MMP-9 using small, interfering, hairpin RNA (siRNA) inhibits glioma cell invasion, angiogenesis and tumor growth. Neuron Glia Biol 1: 165-176, 2004. 